\title{
Downregulation of vascular endothelial growth factor receptor-2 under oxidative stress conditions is mediated by $\beta$-transduction repeat-containing protein via glycogen synthase kinase-3ß signaling
}

\author{
WEN WU, DAIMIN ZHANG, DAORONG PAN, GUANGFENG ZUO, XIAOMIN REN and SHAOLIANG CHEN
}

Department of Cardiology, Nanjing First Hospital, Nanjing Medical University, Qinhuai, Nanjing 210006, P.R. China

Received September 1, 2015; Accepted February 5, 2016

DOI: $10.3892 / \mathrm{ijmm} .2016 .2493$

\begin{abstract}
Vascular endothelial growth factor receptor-2 (VEGFR-2), which is a key determinant of the angiogenecic response, is decreased in diabetic mice under oxidative stress. $\beta$-transduction repeat-containing protein $(\beta$-TrCP) has been reported to participate in VEGFR-2 degradation in thyroid cancer cells. Additionally, glycogen synthase kinase-3 $\beta$ (GSK-3 $\beta$ ) acts as a mediator in the $\beta$-TrCP-induced degradation of several proteins. However, the role played by $\beta$-TrCP and GSK-3 $\beta$ in the degradation of VEGFR-2 in endothelial cells where hyperglycemia had been induced was not fully understood. In the present study, we aimed to analyze the mechanisms of VEGFR-2 degradation by studying excess reactive oxygen species (ROS) induced by hyperglycemia or glucose oxidase (GO). Human umbilical vein endothelial cells (HUVECs) were treated with different concentrations of glucose $(6.6,19.8$ and $33 \mathrm{mM})$, mannitol $(33 \mathrm{mM})$ and $\mathrm{GO}$ (1 U/ml). Angiogenic function, ROS levels, the co-location of VEGFR-2 and $\beta$-TrCP were evaluated. Cells were collected for RT-qPCR and western blot analysis. We noted that angiogenesis was impaired upon increasing the glucose concentration. When HUVECs were in a hyperglycemic state, ROS production increased, comparable to exposure to $\mathrm{GO}$; $\mathrm{GO}$ catalyzes oxidation of glucose into $\mathrm{H}_{2} \mathrm{O}_{2}$ and D-glucono- $\delta$-lactone. Phosphorylated VEGFR-2 was reduced by hyperglycemia while total VEGFR-2 was almost unaltered. However, VEGFR-2 was reduced when directly exposed to ROS, with resultant co-location of $\beta$-TrCP and VEGFR-2. Through a co-immunoprecipitation assay, we noted that ubiquitinated VEGFR-2 was significantly augmented by excess ROS. Decreased VEGFR-2 caused by ROS was ameliorated by $\beta$-TrCP siRNA, proteasome
\end{abstract}

Correspondence to: Professor Shaoliang Chen, Department of Cardiology, Nanjing First Hospital, Nanjing Medical University, 68 Changle Road, Qinhuai, Nanjing 210006, P.R.China

E-mail: chmengx@sina.com

Key words: vascular endothelial growth factor receptor-2, oxidase stress, hyperglycemia, $\beta$-transduction repeat-containing protein, glycogen synthase kinase- $3 \beta$ inhibitor MG132 and GSK-3 $\beta$ activity inhibitor (lithium chloride and SB216763). We suggest that redundant ROS reduces VEGFR-2 through $\beta$-TrCP-mediated VEGFR-2 degradation, which is postulated to be regulated by GSK-3 $\beta$.

\section{Introduction}

Diabetic vasculopathy, a major complication of diabetes, is characteristic of impaired angiogenic response $(1,2)$. Nevertheless, the exact mechanisms have not been thoroughly studied. Previous studies have shown that vascular endothelial growth factor receptor-2 (VEGFR-2), which is expressed mostly in vascular endothelial cells, plays an important role in regulating endothelial cell proliferation, migration and capillary-like tube formation, which are considered to be important components of the angiogenic response (3-5). When stimulated by vascular endothelial growth factor (VEGF), autophosphorylation of VEGFR-2 occurs, leading to angiogenesis $(5,6)$. It has been recently reported that the expression of VEGFR-2 in endothelial cells is decreased in diabetic mice when reactive oxygen species (ROS) is induced by hyperglycemia (7).

The ubiquitin-proteasome system (UPS), one method of protein degradation, is responsible for $80-90 \%$ of proteolysis. There are three enzymes in the UPS, namely ubiquitin-activating enzyme (E1), ubiquitin-conjugating enzyme (E2), and ubiquitin ligase (E3). E1 activates ubiquitin, which is accepted by E2, and E3 targets the protein to which the ubiquitin is attached. The protein is then degraded by the $26 \mathrm{~S}$ proteasome. Thus, E3 is responsible for specificity of protein turnover (8). $\beta$-transduction repeat-containing protein $(\beta-\operatorname{TrCP})$, an F-box component of the Skp1-Cul1-F-box protein ubiquitin ligases which functions as a substrate recognition subunit, has been previously reported to suppress angiogenesis by promoting ubiquitination and degradation of VEGFR-2 in thyroid cancer cells (9-11). However, the role of $\beta$-TrCP in the regulation of VEGFR-2 and angiogenesis in endothelial cells where hyperglycemia is induced is far from $\mathrm{f}$

ully understood. Additionally, $\beta$-TrCP recognizes a consensus sequence of $\operatorname{DSG}(X)_{n} S(X$, any amino acid; $n=2-4)$ in its substrates and requires them to be phosphorylated before regulating degradation (12). Interestingly, the DSG(X) ${ }_{n} S$ motif is present in VEGFR-2 (9). Moreover, as previously demomstrated, glycogen synthase kinase-3 $\beta$ (GSK-3 $\beta$ ) phosphorylates many 
$\beta$-TrCP substrates and mediates their degradation $(9,13,14)$. Accordingly, in the present study we hypothesized that redundant ROS downregulated VEGFR-2 through $\beta$-TrCP-induced UPS-dependent VEGFR-2 degradation, which was likely mediated by GSK-3 $\beta$.

\section{Materials and methods}

Cell culture and glucose or reagent exposure. Human umbilical vein endothelial cells (HUVECs) were obtained from American Type Culture Collection (Manassas, VA, USA). The cells were routinely cultured in Dulbecco's modified Eagle's medium (DMEM) supplemented with $10 \%$ fetal bovine serum (both from Gibco, Shanghai, China) and maintained at $37^{\circ} \mathrm{C}$ in a humidified chamber with $5 \% \mathrm{CO}_{2}$. The culture medium was changed for fresh medium every other day. When they reached $80-90 \%$ confluence after $12 \mathrm{~h}$ of incubation, cells were grouped into various groups: normal glucose $(\mathrm{NG}, 6.6 \mathrm{mM})$, medium glucose (MG, $19.8 \mathrm{mM})$, high glucose ( $\mathrm{HG}, 33 \mathrm{mM})$, mannitol (Man, $33 \mathrm{mM}$ ), SU5416 (1.0 and $10 \mu \mathrm{M}$, respectively, as have been frequently adopted in previous studies) and glucose oxidase (GO, $1 \mathrm{U} / \mathrm{ml}$ ) (15-17). Cells exposed to various concentrations of glucose or Man were incubated for $48 \mathrm{~h}$. Treatment with GO lasted for $15 \mathrm{~min}$, as previously described (7).

Reagents and antibodies. Lipofectamine 2000 and TRIzol reagent were obtained from Invitrogen Life Technologies, Inc. (Grand Island, NY, USA). All the primary antibodies [phospho-VEGFR-2 (Tyr1175) (19A10) rabbit monoclonal antibody (mAb) (\#2478); VEGFR-2 (D5B1) rabbit $\mathrm{mAb}$ (\#9698); $\beta$-TrCP (D13F10) rabbit mAb (\#4394); ubiquitin antibody (\#3933); phospho-GSK-3 $\beta$ (Ser9) (D85E12) rabbit mAb (\#5558); GSK-3 $\beta$ (D5C5Z) rabbit mAb (\#12456)], secondary antibodies [anti-rabbit IgG, HRP-linked antibody (\#7074)], human vascular endothelial growth factor $165\left(\mathrm{hVEGF}_{165}\right)$ and MG132 (a potent proteasome inhibitor) were purchased from Cell Signaling Technology (Danvers, MA, USA). SU5416 (a specific VEGFR-2 inhibitor) and SB216763 (a specific inhibitor of GSK-3 $\beta$ ) was obtained from Tocris Bioscience (Bristol, UK). GO was purchased from Sigma-Aldrich Chemical (St. Louis, USA). $\beta$-TrCP siRNA (sc-37178) and lithium chloride (LiCl) were obtained from Santa Cruz Biotechnology, Inc. (Santa Cruz, CA, USA). $\mathrm{LiCl}$ was used to block GSK-3 $\beta$ activity. A protease inhibitor phenylmethylsulfonyl fluoride (PMSF), electrochemiluminescence (ECL) kit and radio immunoprecipitation assay (RIPA) buffer were purchased from the KeyGen Institute of Biotechnology (Nanjing, China). A PrimeScript First Strand cDNA Synthesis kit and SYBR-Green PCR Master Mix were purchased from Takara Bio (Shiga, Japan).

Cell proliferation assay. Firstly, using a cell counting plate, $5 \times 10^{3}$ HUVECs were seeded into each well of a 96-well plate and counted. The cells were incubated with $\mathrm{NG}, \mathrm{HG}$, Man, SU5416 (1.0 and $10 \mu \mathrm{M}$, respectively) for $48 \mathrm{~h}$ (15-17). Subsequently, $10 \mu \mathrm{l}$ 3-(4,5-dimethylthiazol-2yl)-2,5-diphenyltetrazolium bromide (MTT, $5 \mathrm{mg} / \mathrm{ml}$ ) was added to each well and incubated for $4 \mathrm{~h} ; 100 \mu \mathrm{l}$ formanzan solution was added to wells for an additional $4 \mathrm{~h}$. Absorbance was measured at $570 \mathrm{~nm}$ using a microplate reader (Gene Company Limited, Chai Wan, Hong Kong). The cell proliferation rate (\%) was calculated using the following formula: cell proliferation rate $(\%)=($ OD570, sample - OD570, blank $) /($ OD570, control - OD570, blank) x100.

'Control' refers to the NG group. 'Sample' means other groups in addition to NG group, and 'blank' means a group to which only medium, MTT solution and DMSO were added.

Cell scratch wound-healing assay. A wound healing assay was performed as described previously (18). Once HUVECs at second passage reached approximately $80 \%$ confluence in 6 -well plates, a wound was made with a $10-\mu 1$ pipette tip. The cells were washed with PBS twice and incubated in DMEM with $\mathrm{NG}, \mathrm{MG}, \mathrm{HG}, \mathrm{Man}$ and SU5416 (1.0 and $10 \mu \mathrm{M}$, respectively), as previously described (15). After $24 \mathrm{~h}$ of incubation, cultured cells were photographed and te cell migration activity was quantified by Image J software (version 1.37, National Institutes of Health) by measuring the number of migrated cells in each group.

Capillary-like tube formation assay using Matrigel. According to the manufacturer's instructions, growth factor-reduced basement membrane matrix (BD Matrigel ${ }^{\mathrm{TM}}$; BD Bioscience, Bedford, MA, USA) (300 $\mu \mathrm{l})$ was added to each well of the 24-well plate and gelation at $37^{\circ} \mathrm{C}$ for $0.5 \mathrm{~h}$ was undertaken. HUVECs (1.2x10 ${ }^{5}$ cells) in $300 \mu 1$ DMEM were added to wells coated with Matrigel. The cells were divided into NG, MG, $\mathrm{HG}$, Man and SU5416 (1.0 and $10 \mu \mathrm{M}$, respectively) groups and were incubated at $37^{\circ} \mathrm{C}$ in a humidified chamber of $5 \% \mathrm{CO}_{2}$ for $6 \mathrm{~h}$ to allow for tube formation. Once tube formation images were captured using an inverted microscope (Olympus, Tokyo, Japan), tube structures were quantified by counting branch points in 3 random fields from each well, as described in previous research (19). The average number of branch points was calculated, and each experiment was repeated in triplicate.

MitoSOX ${ }^{T M}$ Red and DAPI. Firstly, at an initial density of $5 \times 10^{3}$ cells/well, the cells seeded into each well of a 96-well plate were incubated with medium containing 6.6 or $33 \mathrm{mM}$ glucose for $48 \mathrm{~h}$, or $1 \mathrm{U} / \mathrm{ml} \mathrm{GO}$ for $15 \mathrm{~min}$. The ROS levels of cells were monitored by MitoSOX Red mitochondrial superoxide indicator (Life Technologies) according to the manufacturer's instructions. The nucleus was stained by DAPI (KeyGen Institute of Biotechnology). Images of MitoSOX Red and DAPI, excited by green light and ultraviolet, were obtained using a fluorescence microscope (Olympus, Tokyo, Japan) and analyzed with ImageJ software.

siRNA transfection. For siRNA transfection, after cells reached $30-50 \%$ confluence they were transfected with scrambled (used as negative control) or $\beta$-TrCP siRNA (both from Santa Cruz Biotechnology, Inc.) using Lipofectamine 2000, according to the manufacturer's instructions. The cells were incubated for $48 \mathrm{~h}$, subsequently exposed to medium containing $1 \mathrm{U} / \mathrm{ml}$ GO for another $15 \mathrm{~min}$ and then the collection of proteins was undertaken.

RNA extraction and reverse transcription-quantitative polymerase chain reaction $(R T-q P C R)$. Total RNA was first extracted from treated HUVECs using TRIzol reagent according to the manufacturer's instructions. In the present study, cDNA was synthesized through reverse transcription and amplification 
using the following primers: VEGFR-2 forward, 5'-TACCT CCACCATGCCAAGTG-3' and reverse, 5'-GATGATTCTG CCСТCCTCCTT-3'; $\beta$-TrCP forward, 5'-AATTCCTCAG AGAGAGAAGACT-3' and reverse, 5'-TCTGGCAAAA CACTAAATATAT-3'; $\beta$-actin forward 5'-TGACGTGGACAT CCGCAAAG-3' and reverse, 5'-CTGGAAGGTGGACA GCGAGG-3'. Amplification was performed according to the following protocol: $95^{\circ} \mathrm{C}$ for $30 \mathrm{sec}$, followed by 40 cycles at $95^{\circ} \mathrm{C}$ for $5 \mathrm{sec}, 60^{\circ} \mathrm{C}$ for $34 \mathrm{sec}$. qPCR was conducted using the ABI 7500 RT-PCR system (Applied Biosystems, Foster City, CA, USA) and SYBR-Green PCR Master Mix, according to the manufacturer's instructions. Relative mRNA expression was calculated using the $2^{-\Delta \Delta \mathrm{Ct}}$ method and presented as foldinduction of the target gene normalized to $\beta$-actin RNA.

Western blot analysis. Total cell lysates were obtained by harvesting the cells in RIPA buffer with a protease inhibitor, phenylmethylsulfonyl fluoride (PMSF), and a phosphatase inhibitor, PhosStop (both purchased from KeyGen Institute of Biotechnology). After incubation at $0^{\circ} \mathrm{C}$ for $20 \mathrm{~min}$, the lysates were scraped and centrifuged at $12,000 \mathrm{x} \mathrm{g}$ at $4^{\circ} \mathrm{C}$ for $20 \mathrm{~min}$. The concentrations of soluble proteins in the supernatants of total cell lysates were determined using a BCA protein assay with bovine serum albumin as the standard. The protein samples containing $50 \mu \mathrm{g}$ total protein were separated on $10 \%$ sodium dodecyl sulfate-polyacrylamide gel electrophoresis (SDS-PAGE) and transferred to PVDF membranes. The membranes were incubated for $2 \mathrm{~h}$ with $5 \%$ skim milk in TBST buffer $(20 \mathrm{~mol} / 1$ Tris- $\mathrm{HCl}, \mathrm{pH} 7.6,150 \mathrm{mmol} / \mathrm{l} \mathrm{NaCl}$, and $0.05 \%$ Tween-20) to block non-specific binding. The membranes were subsequently incubated with specific primary antibodies and gently shaken at $4^{\circ} \mathrm{C}$ overnight, which was followed by incubation with the secondary antibody for $1 \mathrm{~h}$. Immunoreactive protein bands were visualized by chemiluminescence using a Syngene Bio Imaging Device (Syngene, Cambridge, UK).

Confocal microscopy. The co-localization of $\beta$-TrCP and VEGFR-2 was detected by confocal microscopy. HUVECs were incubated with primary antibody for anti- $\beta$-TrCP (Cell Signaling Technology) and anti-VEGFR-2 (Abcam, Cambridge, UK), then with Cy3 goat anti-rabbit antibody and FITC goat anti-mouse. Nuclei were stained with DAPI for $10 \mathrm{~min}$ at room temperature. Cells were imaged using a LSM710 laser scanning confocal microscope (Leica Microsystems, Wetzlar, Germany).

Co-immunoprecipitation assay. In the present study, HUVECs were incubated with $200 \mu$ RIPA buffer and $1 \mu 1$ protease inhibitor at $0^{\circ} \mathrm{C}$ for $1 \mathrm{~h}$, homogenized, and centrifuged at $500 \mathrm{rpm}$ at $4^{\circ} \mathrm{C}$ for $10 \mathrm{~min}$. The supernatant was incubated with VEGFR-2 antibody at a final concentration of $4 \mu \mathrm{g} / \mathrm{ml}$ for each at $4{ }^{\circ} \mathrm{C}$ overnight incubated with $20 \mu$ l Protein G Plus-Agarose (Beyotime, Shanghai, China) and then washed twice with RIPA and protease inhibitor buffer. The immunoprecipitates were collected and eluted from agarose with $30 \mu 1$ SDS-PAGE loading buffer per tube.

Statistical analysis. The data are expressed as the means \pm SD. The student's t-test was employed to compare data between two groups. One-way ANOVA analysis, followed by Dunnett's test, was used to compare multiple groups. In the present study, a P-value $<0.05$ was considered to indicate a statistically significant difference. Moreover, statistical analysis was performed using SPSS 16.0 statistical software (SPSS Inc., Chicago, IL, USA).

\section{Results}

Hyperglycemia and VEGFR-2 inhibitor impair angiogenesis. After exposure for $48 \mathrm{~h}$ to $\mathrm{MG}, \mathrm{HG}$ and SU5416 (a specific VEGFR-2 inhibitor, at 1 and $10 \mu \mathrm{M}$ ), but not Man, the HUVEC proliferation rate was significantly decreased compared with the NG group (Fig. 1A). To further investigate the role of hyperglycemia in inhibiting the angiogenic response, we preformed a cell migration assay. As shown in Fig. 1B, HUVECs exposed to MG, HG and SU5416 exhibited a lower migration rate compared with cells exposed to NG. In the capillary-like tube formation assay, fewer branch points of formed tubes were observed in MG, HG, SU5416 (both in 1 and $10 \mu \mathrm{M})$-stumulated groups, compared with the NG group (Fig. 1C). Taken together, the data in Fig. 1 indicate the role of the impaired VEGF-VEGFR-2 signaling transduction pathway in angiogenesis upon hyperglycemia induction. To investigate the mechanism of the phenomenon above, we subsequently examined the expression level of VEGFR-2 and phosphorylated VEGFR-2 under hyperglycemic conditions.

Hyperglycemia impairs VEGFR-2 activation without markedly affecting its expression. The level of phosphorylated VEGFR-2 was decreased in HUVECs cultured under hyperglycemic conditions for $48 \mathrm{~h}(\mathrm{P}<0.05 \mathrm{vs}$. NG) when activated by VEGF (100 ng/ml for $5 \mathrm{~min}$ ), a specific ligand for VEGFR-2 (Fig. 2A). Although in HUVECs exposed to high glucose the response to VEGF was impaired, total VEGFR-2 protein was not significantly decreased ( $\mathrm{P}>0.05$ vs. NG) (Fig. 2B). Accordingly, the mRNA expression level of VEGFR-2 remained almost unchanged in the HG group ( $\mathrm{P}>0.05$ vs. NG) (Fig. 2C). These findings indicate that high glucose decreases VEGF-induced phosphorylation of VEGFR-2 but has no marked effect on its total expression, both at protein and mRNA levels. In addition, the protein and mRNA levels of $\beta$-TrCP in NG and HG groups were measured. Although $\beta$-TrCP was slightly increased in the HG group, there was no significant difference between the two groups ( $\mathrm{P}>0.05$ vs. NG) (Fig. 2D and E).

VEGFR-2 degradation is induced by ROS overproduction. A previous study has indicated that increased oxidative stress is one of the mechanisms underlying hyperglycemia-induced endothelial dysfunction (20). In the present study, to investigate the role of ROS in regulating VEGFR-2, we employed GO, which catalyzes the oxidation of glucose into $\mathrm{H}_{2} \mathrm{O}_{2}$, in order to create an excess ROS model while excluding the effects of other aspects of diabetic vasculopathy mechanisms. As shown in Fig. 3A, endothelial cells exposed to high glucose for $48 \mathrm{~h}$ exhibited excess ROS production, as detected by MitoSOX Red mitochondrial superoxide indicator. A similar result was also obtained when endothelial cells were treated with $1 \mathrm{U} / \mathrm{ml} \mathrm{GO}$ for $15 \mathrm{~min}$ (Fig. 3A), which concurs with previous research (7). The fluorescence intensity was comparable between the HG and GO group, and it was observed that both were significantly higher than the NG group ( $\mathrm{P}<0.05$ vs. NG). 
A

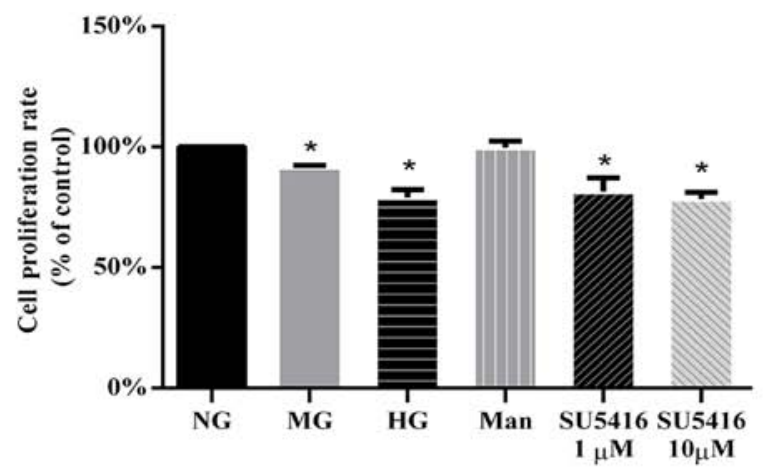

B
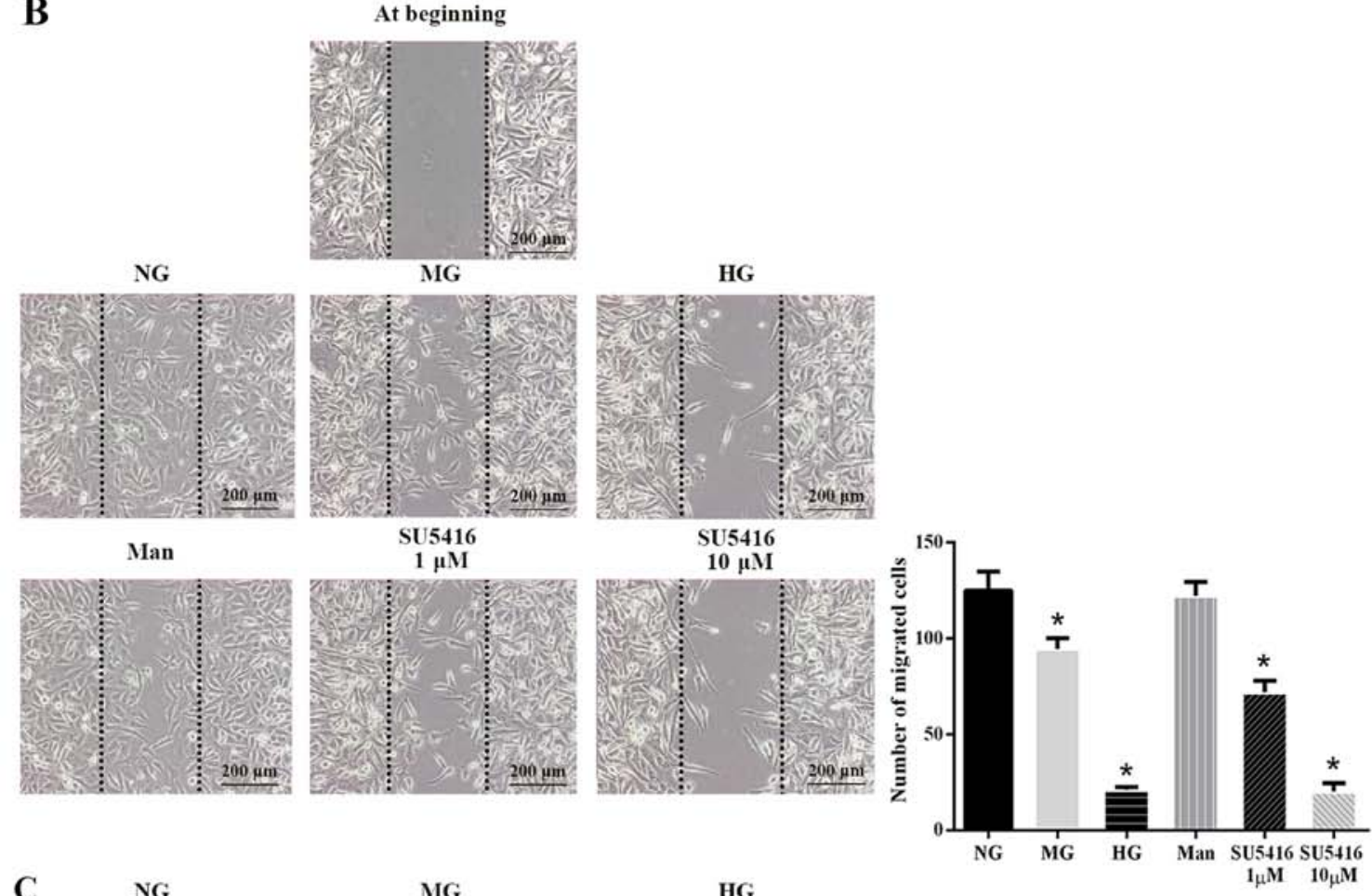

C NG

MG

HG
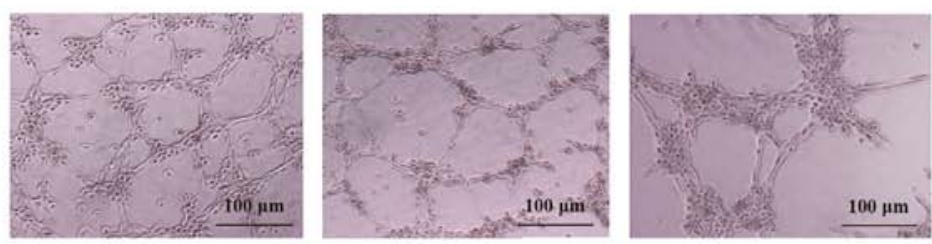

Man

SU5416
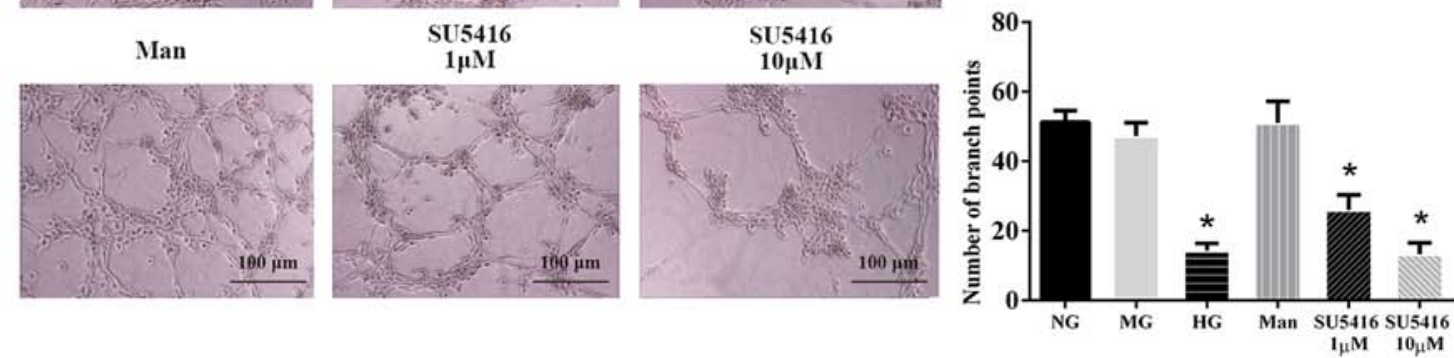

Figure 1. Inhibition of cell proliferation, migration and tube formation in human umbilical vein endothelial cells (HUVECs) exposed to different concentrations of glucose [NG (normal glucose), MG (medium glucose) and HG (high glucose)], mannitol (Man, for osmotic control) and SU5416 (1 and $10 \mu \mathrm{M}$ ). (A) Cells of the six groups were cultured as indicated. Cell viability was determined by MTT assay. Results were presented as the cell proliferation rate described in Materials and methods. (B) Representative images of initial width of the wounded gaps and wound closure in HUVECs that were cultured in DMEM with different treatment after incubation for $24 \mathrm{~h}$. Cell migration activity was quantified by counting the number of migrated cells in each group. (C) Representative images of HUVECs on Matrigel in the different groups. Quantitative analysis of tube formation was undertaken by counting the number of branches from 3 randomly selected fields per well. The results are expressed as the means \pm SD from three independent experiments. "P $<0.05$ vs.NG group.

By contrast to Fig. 2B, VEGFR-2 expression was significantly reduced by GO (P<0.05 vs. GO) (Fig. 3B). Consistent with Fig. 2C, we noted that VEGFR-2 mRNA expression was not markedly altered in the presence of GO, though it was very 
A

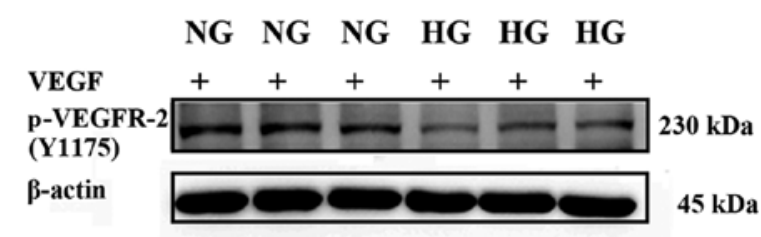

B

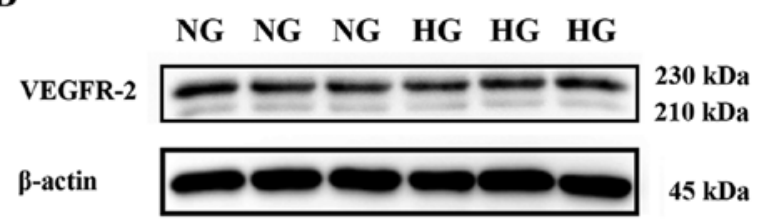

C

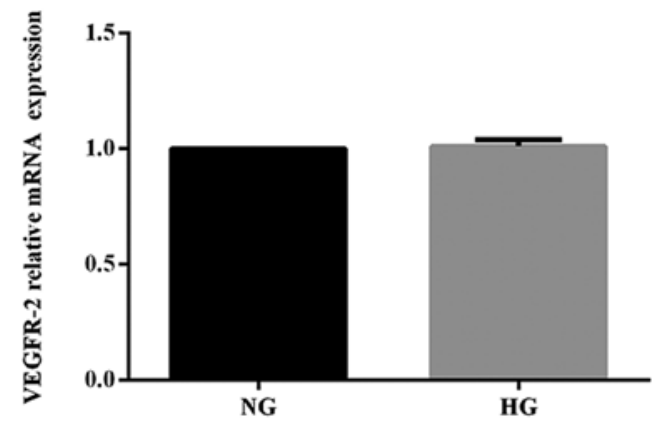

E

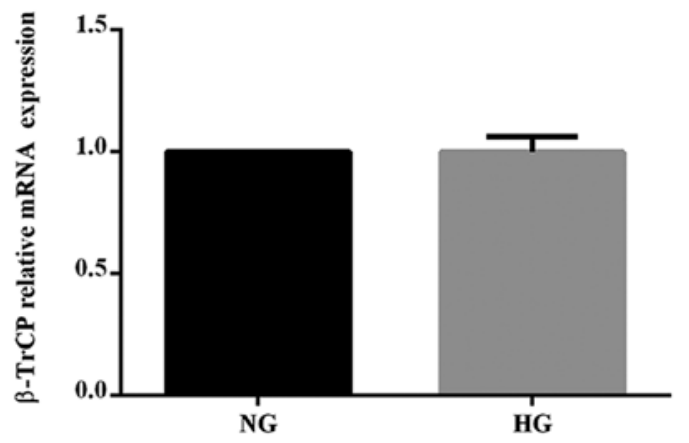

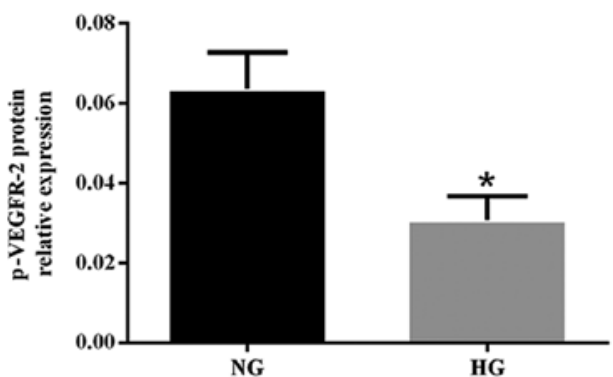

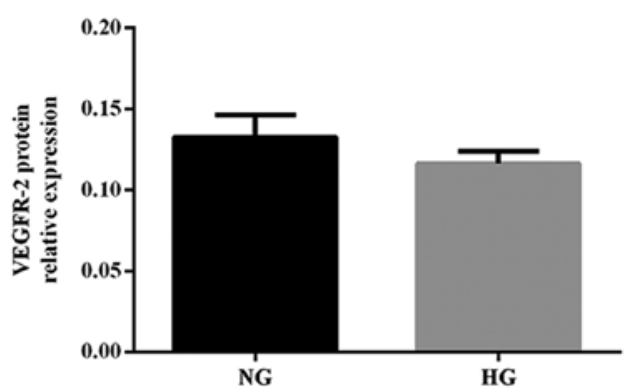

D

NG NG NG HG HG HG

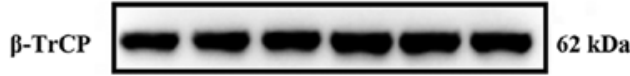

$\beta$-actin
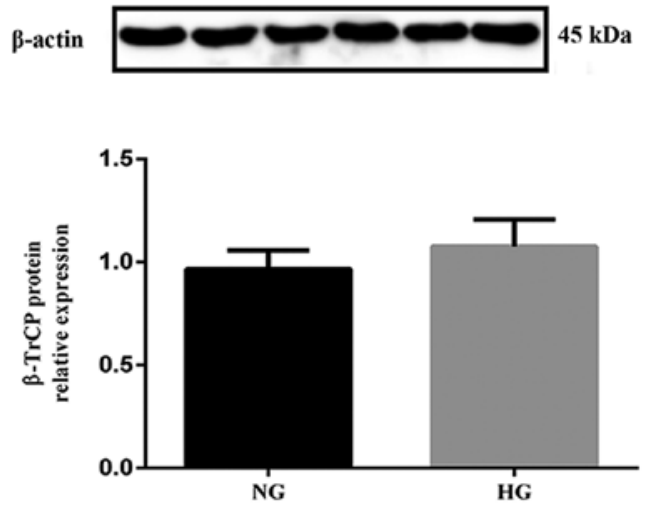

Figure 2. Effect of hyperglycemia on responses of endothelial cells to vascular endothelial growth factor (VEGF), protein and gene expression of VEGF receptor-2 (VEGFR-2) and $\beta$-transduction repeat-containing protein ( $\beta$-TrCP) protein expression. (A) Human umbilical vein endothelial cells (HUVECs) cultured under 6.6 or $33 \mathrm{mM}$ glucose were treated with VEGF. Decreased protein expression of p-VEGFR-2 in HG (high glucose) compared with NG (normal glucose) was noted. Quantification of p-VEGFR-2/ $\beta$-actin. (B and C) Protein and mRNA expression of VEGFR-2 were not significantly altered in HG group compared with NG. (D) Western blot analysis of $\beta$-TrCP protein expression in NG and HG. (E) RT-qPCR analysis of $\beta$-TrCP mRNA expression in NG and HG. The results are expressed as the means $\pm \mathrm{SD}$. ${ }^{*} \mathrm{P}<0.05$ vs NG group.

slightly increased ( $\mathrm{P}>0.05$ vs. GO) (Fig. 3C). Furthermore, the $\beta$-TrCP protein and mRNA expression levels were both assessed. As was clearly demonstrated in Fig. 3D and E, no significant difference between the two groups (NG and GO) was observed ( $\mathrm{P}>0.05$ vs. $\mathrm{GO})$.

Participation of $\beta$-TrCP in the downregulation of VEGFR-2 by $R O S$. Confocal fluorescence microscopy was used to explore whether there was co-location of VEGFR-2 and $\beta$-TrCP. In HUVECs in both the NG and GO groups, cytomembrane expression of VEGFR-2 and cytoplasmic and nuclear expression of $\beta$-TrCP were noted. Moreover, co-localization of VEGFR-2 and $\beta$-TrCP existed both under normal and high ROS conditions. To determine and compare the level of ubiquitinated VEGFR-2 protein in the NG and GO groups, we performed a co-immunoprecipitation assay using anti-VEGFR-2 antibodies, followed by western blot analysis using anti-ubiquitin antibody. There was an increase in VEGFR-2 ubiquitination in endothelial cells exposed to GO (P<0.05 vs. NG) (Fig. 4B). Forty-eight hours after $\beta$-TrCP small interfering RNA (siRNA) $(50 \mathrm{nM})$ was transfected into HUVECs, the $\beta$-TrCP protein level was suppressed $(\mathrm{P}<0.05)$ compared with scrambled siRNA, whereas VEGFR-2 expression was increased $(\mathrm{P}<0.05)$ (Fig. 4C). GO-induced VEGFR-2 

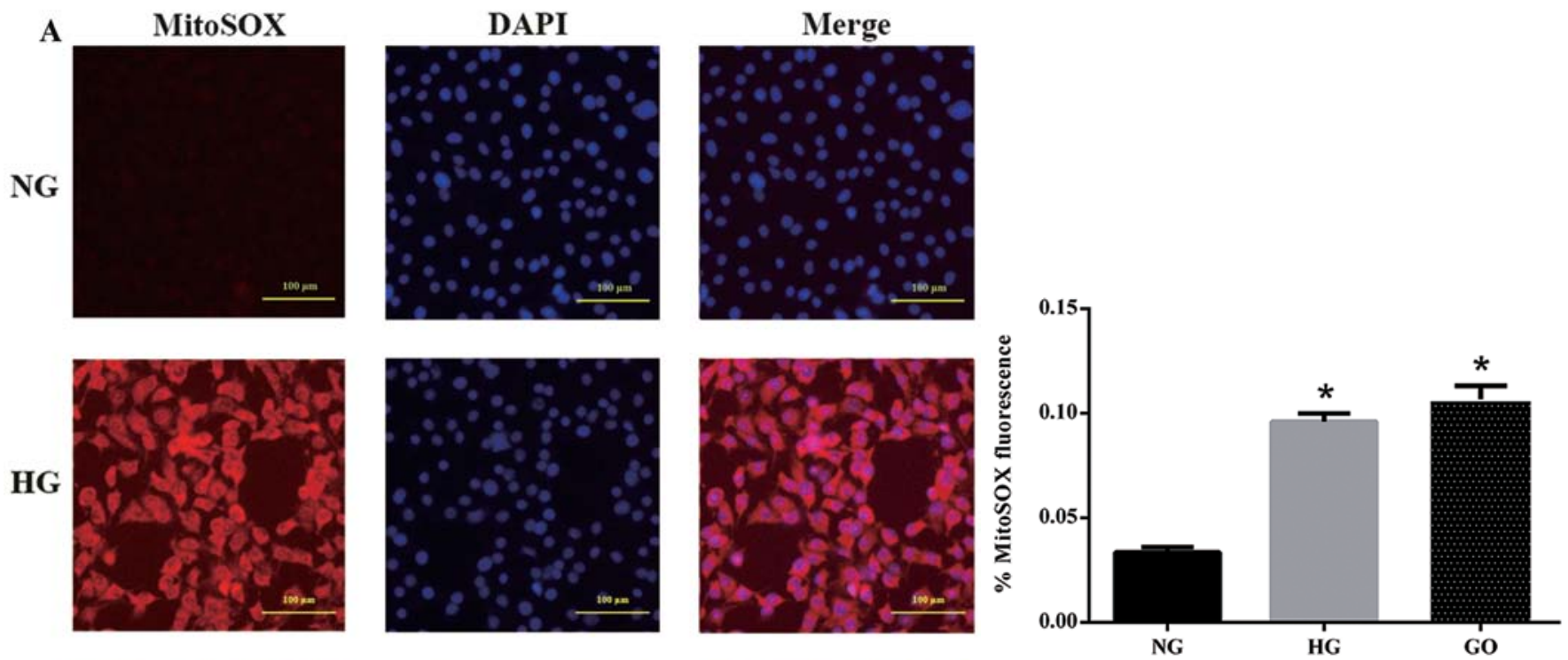

HG
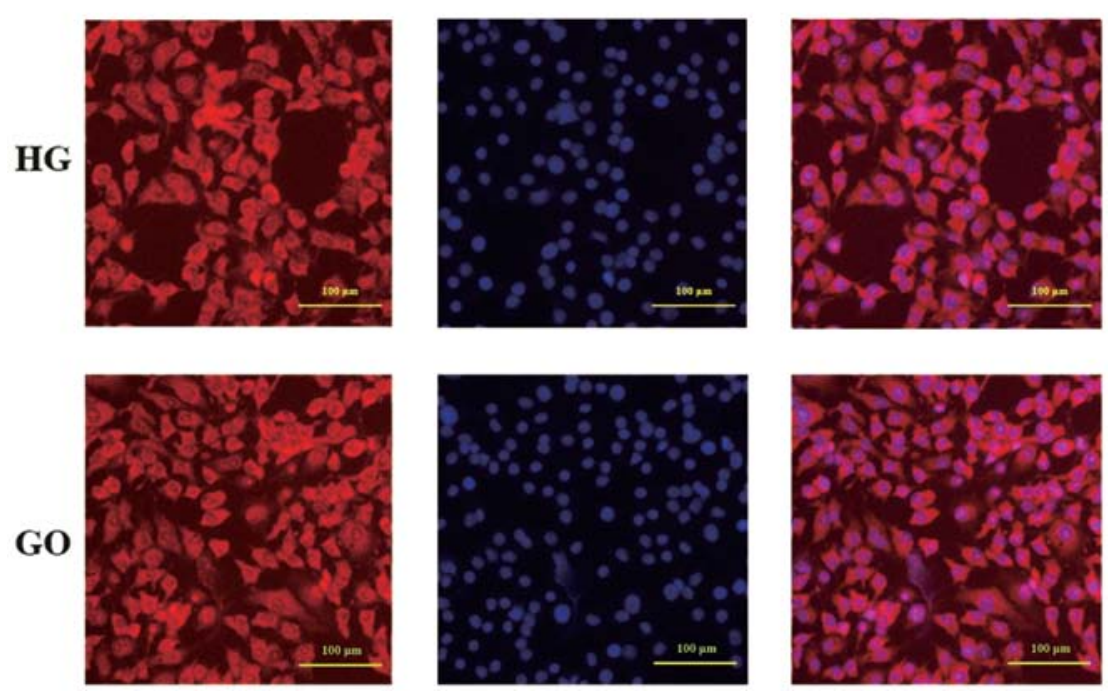

$\mathbf{B}$

NG NG NG GO GO GO
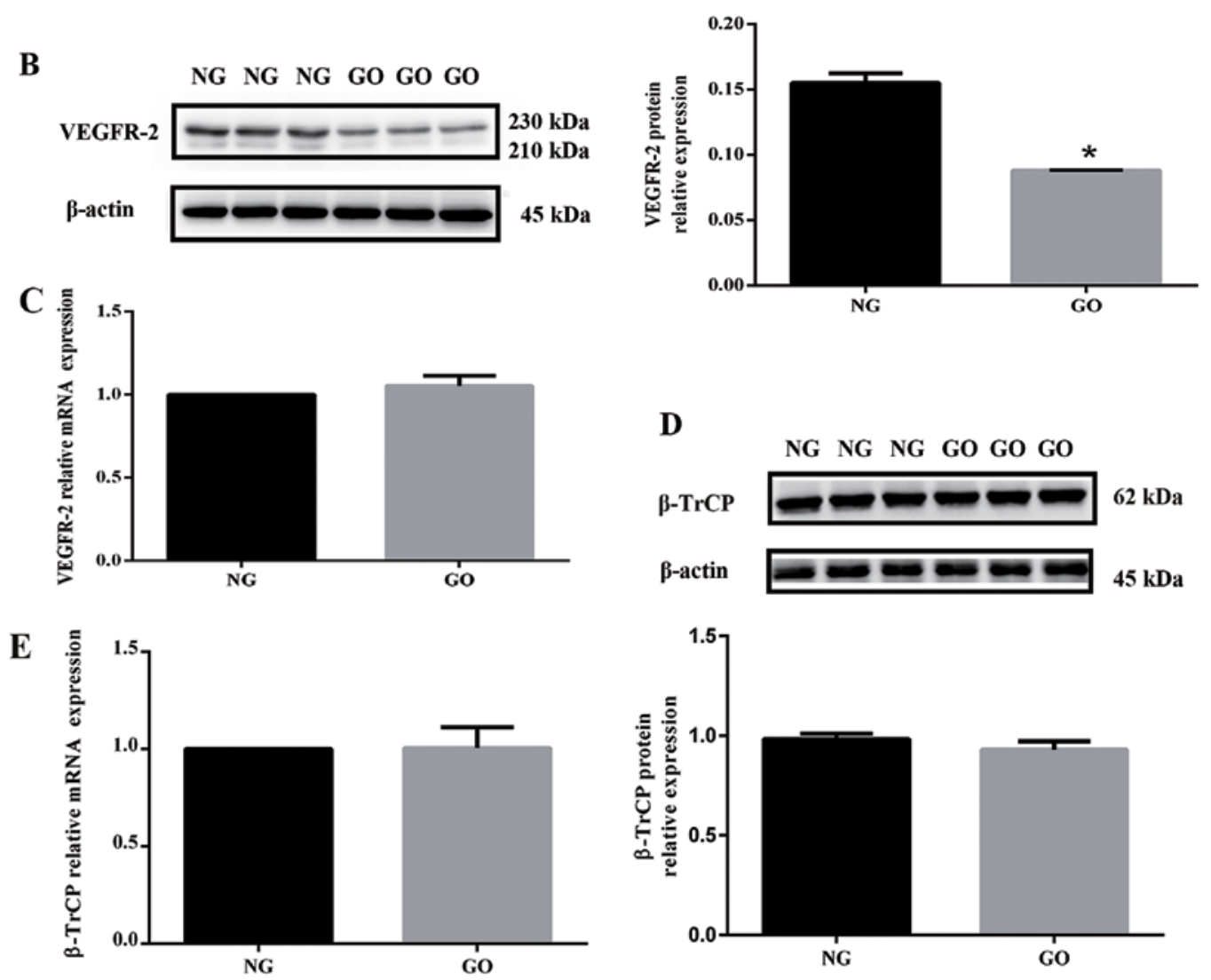

Figure 3. Redundant reactive oxygen species (ROS) production induced by glucose oxidase (GO), which is comparable to hyperglycemic conditions, led to vascular endothelial growth factor receptor-2 (VEGFR-2) degradation but had no marked effect on protein expression of $\beta$-transduction repeat-containing protein ( $\beta$-TrCP). (A) Human umbilical vein endothelial cells (HUVECs) cultured in 6.6 or $33 \mathrm{mM}$ glucose or exposed to GO as indicated were photographed using MitoSOX Red mitochondrial superoxide indicator (red) and DAPI (blue) as a nuclear counterstain. Quantification of relative MitoSOX fluorescence. (B) Decreased protein expression of VEGFR-2 in HUVECs exposed to GO. (C) Relatively unchanged mRNA expression of VEGFR-2 in HUVECs exposed to GO and NG. (D and E) GO exerted no significant effect on the protein level or mRNA level of $\beta$-TrCP in HUVECs. The results are expressed as the means $\pm \mathrm{SD}$. $\mathrm{P}<0.05$ vs.normal glucose $(\mathrm{NG})$ group. 
A VEGFR-2
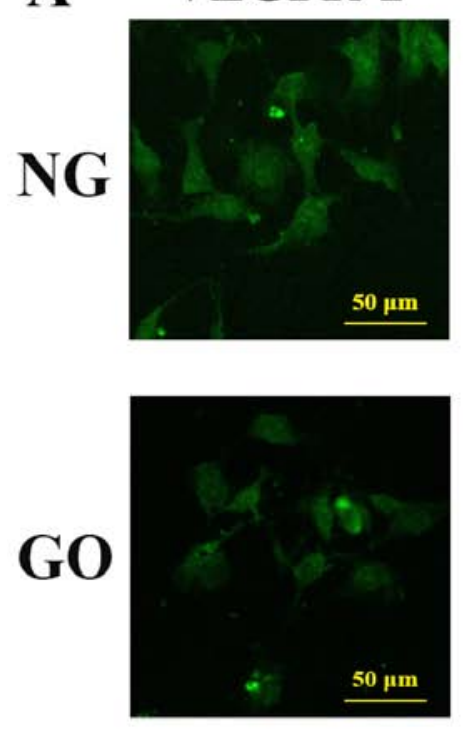

$\beta-\operatorname{TrCP}$
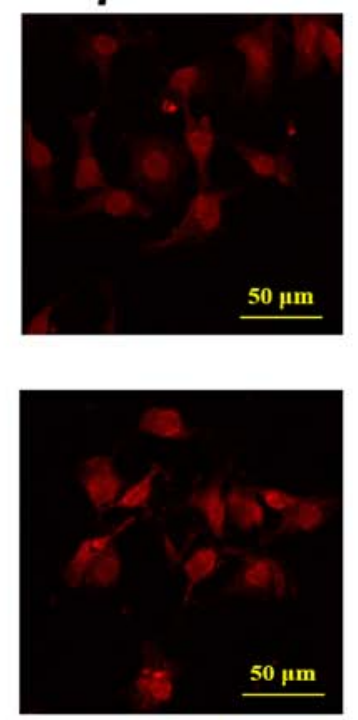

DAPI
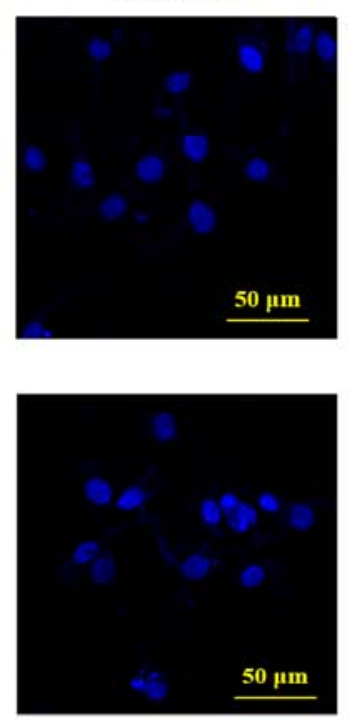

Merge
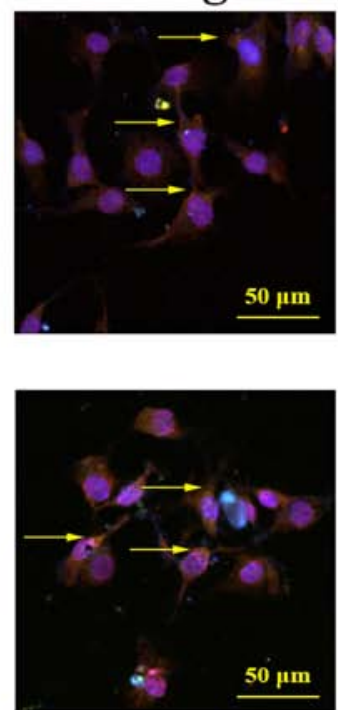

B
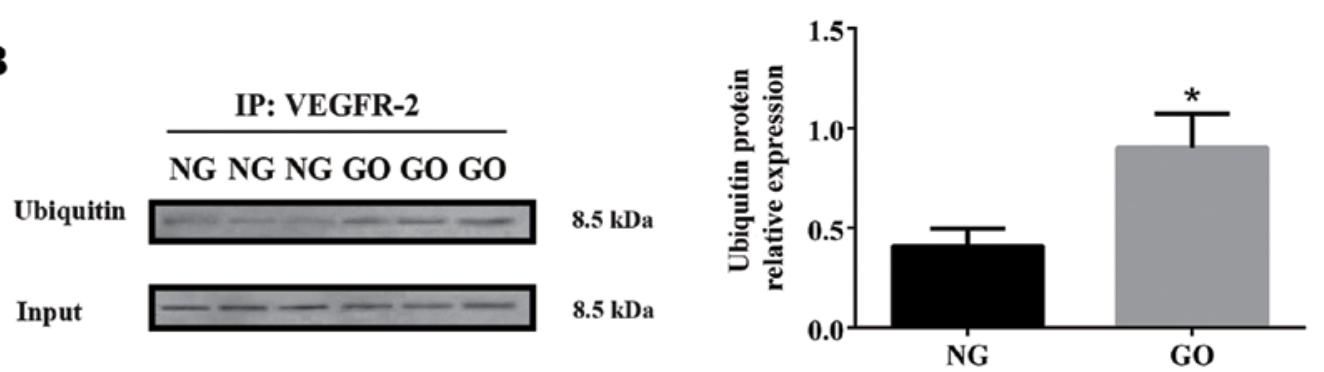

C
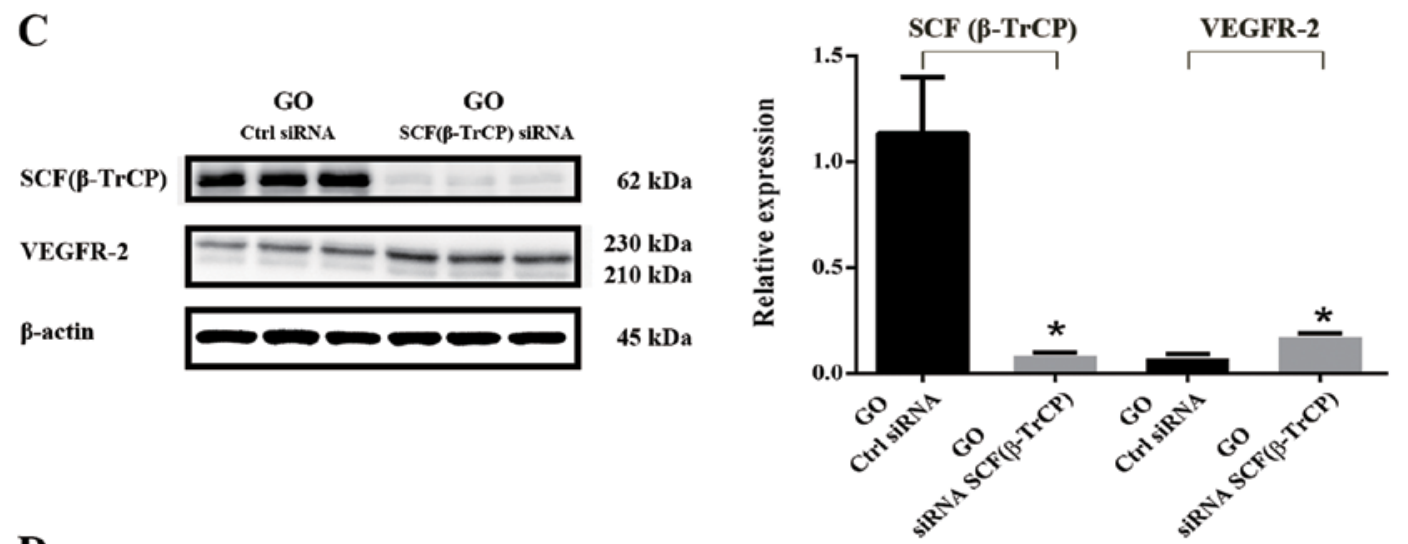

D

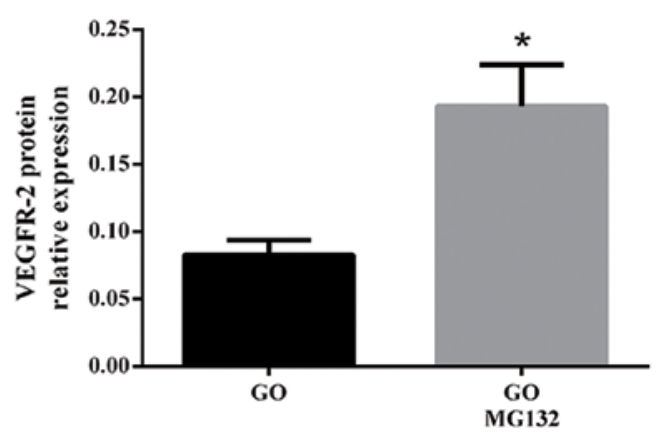

Figure 4. Reactive oxygen species (ROS) induces interaction of vascular endothelial growth factor receptor-2 (VEGFR-2) and $\beta$-transduction repeat-containing protein $(\beta$-TrCP), and suppresses $\beta$-TrCP-ubiquitin proteasome system-induced VEGFR-2 upon glucose oxidase (GO) exposure. (A) Intracellular locations of VEGFR-2 and $\beta$-TrCP were detected by confocal immunofluorescence microscopy. Green, VEGFR-2; red, $\beta$-TrCP; blue, nuclear staining with DAPI. Yellow arrows in Merge panels indicate co-localization of VEGFR-2 and $\beta$-TrCP. (B) Immunoprecipitates of anti-VEGFR-2 antibody against human umbilical vein endothelial cell (HUVEC) lysates were resolved and blotted against anti-ubiquitin antibody. Quantification of ubiquitin/input. (C) $\beta$-TrCP siRNA suppressed $\beta$-TrCP expression but enhanced VEGFR-2 expression in HUVECs exposed to GO. (D) VEGFR-2 protein expression in HUVECs cultured with GO after treatment with $10 \mu \mathrm{M}$ MG132 (4 h) was increased compared with GO treatment alone. The results are expressed as the means \pm SD. *P $<0.05$ vs. normal glucose (NG) group. 
A

\author{
The DSG(X) $)_{\mathbf{n}}$ S Motif
}

$\begin{array}{ll}\text { Homo } & 1181-S E T L S M E E D S G L S L P T S P V S C M E E E \text { ECDP-1230 } \\ \text { Mus } & 1181-\text { TLSMEEDSGLSLPTSPVSCMEEEEVCDPKF-1230 } \\ \text { Rattus } & 1181-\text { SMEEDSGLSLPTSPVSCMEEEVCDPKFH-1230 } \\ \text { Coturnix } & 1181-\text { ESGLSLPTSPASCKEEEEVCDPKFHDNT-1230 }\end{array}$

B NG NG NG GO GO GO

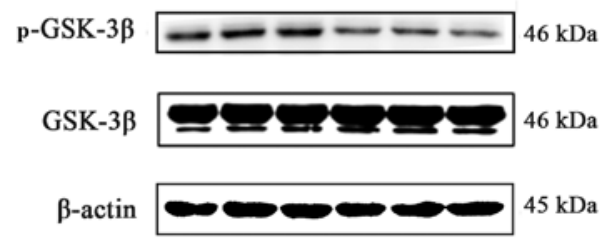

C
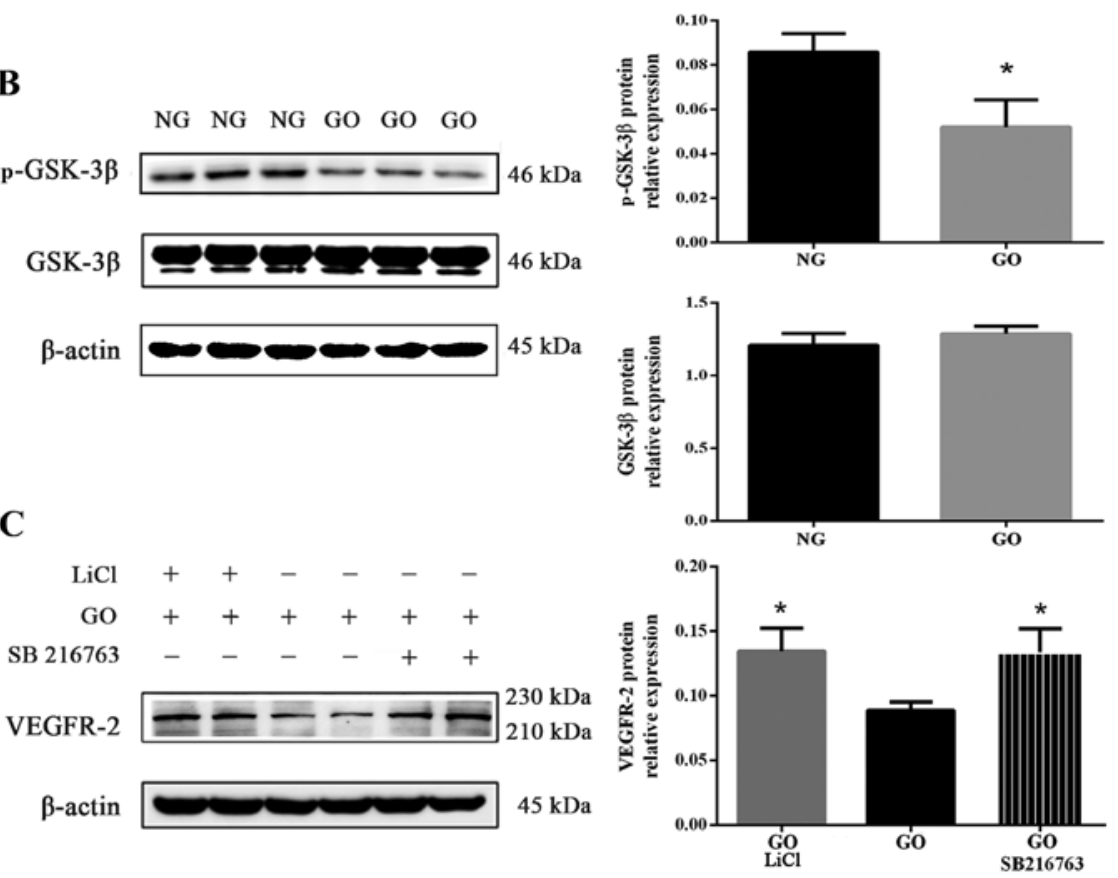

Figure 5. Reserved DSG $(\mathrm{X})_{n} \mathrm{~S}$ motif provides a binding site for $\beta$-transduction repeat-containing protein $(\beta-\operatorname{TrCP})$, and activated glycogen synthase kinase-3 $\beta$ (GSK-3 $\beta$ ) mediates this process. (A) Sequence alignment of human, mouse, rat and coturnix vascular endothelial growth factor receptor-2 (VEGFR-2) cDNA showed a conserved DSG(X) $)_{n}$ S binding motif. (B) Reduced GSK-3 $\beta$ phosphorylation with almost unaltered total GSK-3 $\beta$ in human umbilical vein endothelial cells (HUVECs) exposed to glucose oxidase (GO). (C) Pretreatment for 30 min with LiCl (20 $\mu \mathrm{M})$ or SB216763 (20 $\mu \mathrm{M})$ enhanced the protein expression of VEGFR-2 in HUVECs cultured with GO. The results are expressed as the means \pm SD. ${ }^{*}<<0.05$ vs. normal glucose (NG) group.

degradation was abolished by a 4-h treatment with $10 \mu \mathrm{M}$ MG132, a proteasome inhibitor $(\mathrm{P}<0.05)$ (Fig. 4D).

ROS-activated GSK-3 $\beta$ induces VEGFR-2 degradation in $H U V E C s$. Sequence alignment of the $\operatorname{DSG}(\mathrm{X})_{\mathrm{n}} \mathrm{S}$ binding motif was obtained using the Database of the National Center for Biotechnology Information. The $\operatorname{DSG}(\mathrm{X})_{\mathrm{n}} \mathrm{S}$ motif is well conserved in VEGFR-2 across many species, including humans, mice, rats and coturnix (Fig. 5A). After 15 min incubation with $1 \mathrm{U} / \mathrm{ml} \mathrm{GO}$, GSK-3 $\beta$ phosphorylation (Serine 9) was attenuated $(\mathrm{P}<0.05$ vs. $\mathrm{NG})$ but total GSK-3 $\beta$ protein expression was almost unchanged (Fig. 5B). As is well known, phosphorylated GSK-3 $\beta$ acts as an inactivated form, and decreased phosphorylated GSK-3 $\beta$ upon exposure to GO, as shown in Fig. 5B, was noted; this signified that GSK-3 $\beta$ was enhanced by excess ROS.

In this study, to verify whether activated GSK-3 $\beta$ was involved in the degradation process of VEGFR-2, we exposed HUVECs for $30 \mathrm{~min}$ to GSK-3 $\beta$ inhibitor, $\mathrm{LiCl}(20 \mathrm{mM})$ and SB216763 (20 mM), before exposure to GO, as also previously described (21). We noted that VEGFR-2 protein expression of cells co-cultured with GO and GSK-3 $\beta$ inhibitors $(\mathrm{LiCl}$ and SB216763) increased compared with the GO group $(\mathrm{P}<0.05)$ (Fig. 5C). These findings demonstrate that GSK-3 $\beta$ plays a key role in regulating VEGFR-2 degradation.

\section{Discussion}

VEGFR-2 plays an important role in the angiogenic response (3-5). In the present study, we demonstrated the impairment of angiogenic function in endothelial cells when exposed to VEGFR-2 inhibitor (SU5416) as well as high glucose stimulation. The protein expression of VEGFR-2 was not significantly altered in the HG group, whereas it was significantly decreased after GO exposure (Figs. 2B and 3B). Warren et al have reported that stimulation with high glucose for $48 \mathrm{~h}$ results only in decreased abundance of plasma membrane-localized VEGFR-2. As noted in the present study, ROS production was induced under hyperglycemic conditions, and we suggest that downregulation of membrane VEGFR-2 expression occurred due to disruption of cytoplasm trafficking from Golgi apparatus to the membrane. The total protein level of VEGFR-2 is significantly altered only when the Golgi-localized pool of VEGFR-2 is decreased under conditions such as chronic exposure to hyperglycemia and ROS, rather than a relatively transient exposure to hyperglycemia (7). Moreover, we suggest that the impaired angiogenic response in the short term was partially due to downregulation of the phosphorylated VEGFR-2 (Threonine 1175), leading to inhibition of the VEGF-VEGFR-2 signaling pathways (Fig. 2A).

GSK-3 $\beta$ acts as a key point for convergent insulin signaling pathways in endothelial cells to angiogenic responses; the 
enzyme is a downstream target of PI3K/Akt signaling pathway and is inactivated by phosphorylation (22). Previous studies have noted that GSK-3 $\beta$ activity is upregulated in the skeletal muscle of T2DM patients and in diabetic mice, indicating that activated GSK-3 $\beta$ causes dysfunction of insulin signaling and then diabetes $(23,24)$. SB216763, a specific inhibitor of GSK-3 $\beta$, does not affect other relevant protein kinase, including PDK-1 and PKB (25). Another inhibitor, LiCl, has been reported to exert various effects on other protein kinases, while in the present study, upon exposure to $\mathrm{GO} \mathrm{LiCl}$ clearly ameliorated VEGFR-2 degradation just as SB216763 also did $(26,27)$. Moreover, $\mathrm{LiCl}$ is known to stimulate glucose uptake, glycogen synthesis and normalize insulin sensitivity in diabetic rats (28). Clinical results have also demonstrated that $\mathrm{LiCl}$ improved impaired wound healing in diabetic patients by inducing the Wnt signaling pathway $(29,30)$. The data of the present study indicated that GSK-3 $\beta$ expression was slightly enhanced and subsequently induced the degradation of VEGFR-2. The insulin receptor signaling is diminished by ROS (31). However, whether $\beta$-TrCP is directly regulated by GSK-3 $\beta$ has not yet been explored.

$\beta$-TrCP, an F-box component of the Skp1-Cul1-F-box protein ubiquitin ligases, functioning as a substrate recognition subunit, has been reported to suppress angiogenesis by promoting ubiquitination and degradation of VEGFR-2 in thyroid cancer cells (9-11). Our results showed that VEGFR-2 and $\beta$-TrCP were co-located in the cytomembrane under high GO conditions. We noted that $\beta$-TrCP protein expression in HUVECs was suppressed by $\beta$-TrCP siRNA, whereas VEGFR-2 expression increased. GSK-3 $\beta$ acts as a mediator in the degradation of several proteins induced by $\beta$-TrCP $(13,14)$. Further experiments should be undertaken to determine whether $\beta$-TrCP and GSK-3 $\beta$ play roles in the degradation of VEGFR-2 in endothelial cells in which hyperglycemia has been induced. Moreover, we noted that the degradation of VEGFR-2 induced by GO was abolished by MG132, a proteasome inhibitor, as well as by $\mathrm{LiCl}$. GO attenuated GSK-3 $\beta$ phosphorylation in HUVECs whereas the protein expression of GSK-3 $\beta$ and $\beta$-TrCP was not significantly altered upon exposure to GO. GO catalyzes the oxidation of D-glucose to produce gluconic and hydrogen peroxide $\left(\mathrm{H}_{2} \mathrm{O}_{2}\right)$ (7). In this study, we noted that $\mathrm{H}_{2} \mathrm{O}_{2}$ induced by high glucose stimulation impaired VEGF-2 function and decreased $\mathrm{p}-\mathrm{GSK}-3 \beta$, but not $\beta$-TrCP. The results suggest that the downregulation of VEGFR-2 induced by $\beta$-TrCP depends on GSK-3 $\beta$ activity.

Certain VEGFR-2 phosphorylation sites are responsible for ubiquitination and degradation, such as Serine 1188/Serine 1191 (Ser 1188/Ser 1191) (32); according to this study by Meyer et al, the phosphorylation of Ser $1188 / \operatorname{Ser} 1191$ is upregulated, which mediates the degradation of VEGFR-2. Taking this into consideration, we predict that GSK-3 $\beta$ phosphorylated VEGFR-2 at sites within the DSG(X) $)_{n}$ S motif.

Our results demonstrate that reduction of VEGFR-2 under hyperglycemic conditions induced by excess ROS was regulated by GSK-3 $\beta$ coupling with $\beta$-TrCP. We suggest that GSK-3 $\beta$ inhibitors act as a promising therapeutic target. In conclusion, these findings provide novel insights into the mechanisms of vascular pathophysiology and may potentially be used in the clinical treatment of diabetes mellitus.

\section{Acknowledgements}

The present study was supported by grants from the National Natural Scientific Foundation of China (nos. 81270191 and 81370304).

\section{References}

1. Waltenberger J: VEGF resistance as a molecular basis to explain the angiogenesis paradox in diabetes mellitus. Biochem Soc Trans 37: 1167-1170, 2009.

2. Schiekofer S, Galasso G, Sato K, Kraus BJ and Walsh K: Impaired revascularization in a mouse model of type 2 diabetes is associated with dysregulation of a complex angiogenic-regulatory network. Arterioscler Thromb Vasc Biol 25: 1603-1609, 2005.

3. Shibuya M: Vascular endothelial growth factor and its receptor system: physiological functions in angiogenesis and pathological roles in various diseases. J Biochem 153: 13-19, 2013.

4. Shibuya M: VEGFR and type-V RTK activation and signaling. Cold Spring Harb Perspect Biol 5: a009092, 2013.

5. Koch S and Claesson-Welsh L: Signal transduction by vascular endothelial growth factor receptors. Cold Spring Harb Perspect Med 2: a006502, 2012.

6. Shibuya M and Claesson-Welsh L: Signal transduction by VEGF receptors in regulation of angiogenesis and lymphangiogenesis. Exp Cell Res 312: 549-560, 2006.

7. Warren CM, Ziyad S, Briot A, Der A and Iruela-Arispe ML: A ligand-independent VEGFR2 signaling pathway limits angiogenic responses in diabetes. Sci Signal 7: ra1, 2014.

8. Chen YS and Qiu XB: Ubiquitin at the crossroad of cell death and survival. Chin J Cancer 32: 640-647, 2013.

9. Shaik S, Nucera C, Inuzuka H, Gao D, Garnaas M, Frechette G, Harris L, Wan L, Fukushima H, Husain A, et al: SCF( $\beta$-TRCP) suppresses angiogenesis and thyroid cancer cell migration by promoting ubiquitination and destruction of VEGF receptor 2 . J Exp Med 209: 1289-1307, 2012.

10. Wei S, Lin LF, Yang CC, Wang YC, Chang GD, Chen H and Chen CS: Thiazolidinediones modulate the expression of betacatenin and other cell-cycle regulatory proteins by targeting the F-box proteins of Skp1-Cul1-F-box protein E3 ubiquitin ligase independently of peroxisome proliferator-activated receptor gamma. Mol Pharmacol 72: 725-733, 2007.

11. Wei S, Yang HC, Chuang HC, Yang J, Kulp SK, Lu PJ, Lai MD and Chen CS: A novel mechanism by which thiazolidinediones facilitate the proteasomal degradation of cyclin D1 in cancer cells. J Biol Chem 283: 26759-26770, 2008.

12. Cardozo T and Pagano M: The SCF ubiquitin ligase: insights into a molecular machine. Nat Rev Mol Cell Biol 5: 739-751, 2004.

13. Li X, Liu J and Gao T: beta-TrCP-mediated ubiquitination and degradation of PHLPP1 are negatively regulated by Akt. Mol Cell Biol 29: 6192-6205, 2009.

14. Liu C, Li Y, Semenov M, Han C, Baeg GH, Tan Y, Zhang Z, Lin $\mathrm{X}$ and He X: Control of beta-catenin phosphorylation/degradation by a dual-kinase mechanism. Cell 108: 837-847, 2002.

15. Bischof M, Abdollahi A, Gong P, Stoffregen C, Lipson KE, Debus JU, Weber KJ and Huber PE: Triple combination of irradiation, chemotherapy (pemetrexed), and VEGFR inhibition (SU5416) in human endothelial and tumor cells. Int J Radiat Oncol Biol Phys 60: 1220-1232, 2004.

16. Yang BR, Hong SJ, Lee SM, Cong WH, Wan JB, Zhang ZR, Zhang QW, Zhang Y, Wang YT and Lin ZX: Pro-angiogenic activity of notoginsenoside R1 in human umbilical vein endothelial cells in vitro and in a chemical-induced blood vessel loss model of zebrafish in vivo. Chin J Integr Med: Dec 22, 2014 (Epub ahead of print).

17. Cukiernik M, Hileeto D, Evans T, Mukherjee S, Downey D and Chakrabarti S: Vascular endothelial growth factor in diabetes induced early retinal abnormalities. Diabetes Res Clin Pract 65: 197-208, 2004.

18. Ettenson DS and Gotlieb AI: Centrosomes, microtubules, and microfilaments in the reendothelialization and remodeling of double-sided in vitro wounds. Lab Invest 66: 722-733, 1992.

19. Pollman MJ, Naumovski L and Gibbons GH: Endothelial cell apoptosis in capillary network remodeling. J Cell Physiol 178: 359-370, 1999.

20. Giacco F and Brownlee M: Oxidative stress and diabetic complications. Circ Res 107: 1058-1070, 2010. 
21. Choi SE, Jang HJ, Kang Y, Jung JG, Han SJ, Kim HJ, Kim DJ and Lee KW: Atherosclerosis induced by a high-fat diet is alleviated by lithium chloride via reduction of VCAM expression in ApoE-deficient mice. Vascul Pharmacol 53: 264-272, 2010.

22. Kobayashi T, Matsumoto T and Kamata K: The PI3-K/Akt pathway: roles related to alterations in vasomotor responses in diabetic models. J Smooth Muscle Res 41: 283-302, 2005.

23. Nikoulina SE, Ciaraldi TP, Mudaliar S, Mohideen P, Carter L and Henry RR: Potential role of glycogen synthase kinase-3 in skeletal muscle insulin resistance of type 2 diabetes. Diabetes 49: 263-271, 2000.

24. Eldar-Finkelman H and Krebs EG: Phosphorylation of insulin receptor substrate 1 by glycogen synthase kinase 3 impairs insulin action. Proc Natl Acad Sci USA 94: 9660-9664, 1997.

25. Coghlan MP, Culbert AA, Cross DA, Corcoran SL, Yates JW, Pearce NJ, Rausch OL, Murphy GJ, Carter PS, Roxbee Cox L, et al: Selective small molecule inhibitors of glycogen synthase kinase-3 modulate glycogen metabolism and gene transcription. Chem Biol 7: 793-803, 2000.

26. Mora A, Sabio G, Risco AM, Cuenda A, Alonso JC, Soler G and Centeno F: Lithium blocks the PKB and GSK3 dephosphorylation induced by ceramide through protein phosphatase- $2 \mathrm{~A}$. Cell Signal 14: 557-562, 2002.
27. Klein PS and Melton DA: A molecular mechanism for the effect of lithium on development. Proc Natl Acad Sci USA 93: 8455-8459, 1996.

28. Rossetti L: Normalization of insulin sensitivity with lithium in diabetic rats. Diabetes 38: 648-652, 1989.

29. Dejana E: The role of wnt signaling in physiological and pathological angiogenesis. Circ Res 107: 943-952, 2010.

30. Qi W, Yang C, Dai Z, Che D, Feng J, Mao Y, Cheng R, Wang Z, He X, Zhou T, et al: High levels of pigment epithelium-derived factor in diabetes impair wound healing through suppression of Wnt signaling. Diabetes 64: 1407-1419, 2015.

31. Hou Q, Lei M, Hu K and Wang M: The effects of high glucose levels on reactive oxygen species-induced apoptosis and involved signaling in human vascular endothelial cells. Cardiovasc Toxicol 15: 140-146, 2015.

32. Meyer RD, Srinivasan S, Singh AJ, Mahoney JE, Gharahassanlou KR and Rahimi N: PEST motif serine and tyrosine phosphorylation controls vascular endothelial growth factor receptor 2 stability and downregulation. Mol Cell Biol 31: 20102025,2011 